\title{
Light, Bias, and Temperature Effects on Organic TFTs
}

\author{
N. Wrachien, A. Cester, N. Bellaio, A. Pinato, M. Meneghini, A. Tazzoli, G. Meneghesso \\ Department of Information Engineering \\ University of Padova \\ Via Gradenigo 6B, Padova - Italy \\ phone: +39-0498277625, fax: +39-0498277699; e-mail: wrachien@dei.unipd.it
}

\author{
K. Myny, S. Smout, J. Genoe \\ imec \\ Kapeldreef 75, 3001 Leuven, Belgium
}

\begin{abstract}
In this work we present the instabilities observed in organic Thin-Film Transistors when subjected to stress test in different bias, temperature and illumination conditions. $\mathrm{C}-\mathrm{V}$ measurements, indicate the presence of two distinct trapping phenomena. Appreciable charge trapping can be achieved using relatively high biases for long times (1000s). Illumination strongly enhances charge trapping only under positive gate biases, while it has no effect on the charge trapping/detrapping under negative gate bias. Charge detrapping is thermally activated coherently with trapping/detrapping at the $\mathrm{SiO}_{2} /$ pentacene interface from hydrogenoid species. A first order model explaining the observed relaxation kinetics is also presented.
\end{abstract}

Keywords-component; Organic thin film transistors, TFT, organic electronics, charge trapping, traps, reliability.

\section{INTRODUCTION}

Organic thin-film transistors (OTFT) are attracting much attention due to their recent performance improvements [1]-[4]. In particular, many publications reported very good results in terms of mobility, subthreshold slope, and $\mathrm{I}_{\mathrm{ON}} / \mathrm{I}_{\mathrm{OFF}}$ ratio, making OTFTs a very compelling candidate as possible replacement for their inorganic counterparts in some specific applications. As a representative example, since their first appearances in the literature, OTFTs gained several orders of magnitude in terms of carrier mobility [5]-[8]. OTFTs favorably compare with inorganic thin-film transistor due to their much lower cost and ease of manufacture. In fact, many organic semiconductors can be deposited at very low temperatures, eliminating the need for very high and unpractical thermal budgets [9]-[10]. In addition organic semiconductor deposition techniques are well suited for large area devices, such as LCD or AMOLED devices. Another advantage of organic semiconductors over their inorganic counterpart, is the compatibility with many plastic substrates, which are flexible, much more robust, inexpensive and much lighter than glass. This latter advantage, would allow a further reduction of the finished device cost and it would open the door to a broad field of applications, which can be hardly achieved using inorganic semiconductors, such as flexible displays,

This work was partially supported by Progetto di Ateneo 2009 - Università di Padova, Italy (Project Number CPDA083941). smart textiles, or various kind of disposable electronic devices like smart labels or cheap lab-on-a-chip.

Unfortunately, many issues are still affecting OTFTs, such as the sensitivity to external environment factors (temperature, humidity, light) [11]-[12], the poor stability and the shorter lifetime if compared to silicon TFTs.

Many publications reported the most recent advancements on new structures, materials or deposition techniques for organic transistors [5]-[10]. However, still few publications have addressed the stability of the electrical characteristics of OTFTs subjected to bias [13]-[16]. The bias stability is an issue in several applications, but it is crucial especially for AMOLED displays [17]-[22]. From this application viewpoint, the light sensitivity is also a concern, because it might enhance the bias effects. Moreover, even the temperature might enhance the effects of bias. At this regard, very few authors in the literature explored the effects of temperature and bias on the stability of organic thin film transistors.

The aim of this work is to investigate the effects of bias combined with illumination and temperature on the OTFT electrical characteristics. We also provide a first order model, which allows to determine the energetic trap density distribution.

\section{EXPERIMENTAL AND DEVICES}

Throughout this work, we analyzed p-type bottom-contact bottom-gate pentacene OTFTs (see Fig. 1a). The gate dielectric

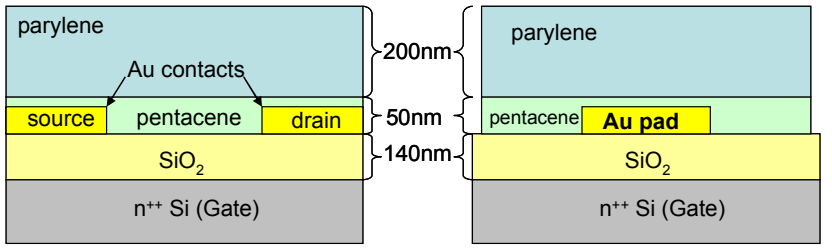

(a)

(b)

Figure 1. Cross section of the devices used throughout this work: (a) the organic thin-film-transistor; (b) the organic capacitor. 
consists of a $140-\mathrm{nm}$ thermal $\mathrm{SiO}_{2}$ layer grown over an $\mathrm{n}^{++}$ silicon substrate, which also acts as gate contact. A 50-nm pentacene layer was deposited by evaporation. Source and drain contacts are made of 30-nm gold layer. The gold layer was deposited by sputtering and patterned using a standard liftoff technique. A $200-\mathrm{nm}$ parylene layer is employed as encapsulation layer and it has been deposited using chemical vapor deposition at a temperature below $40^{\circ} \mathrm{C}$. The devices

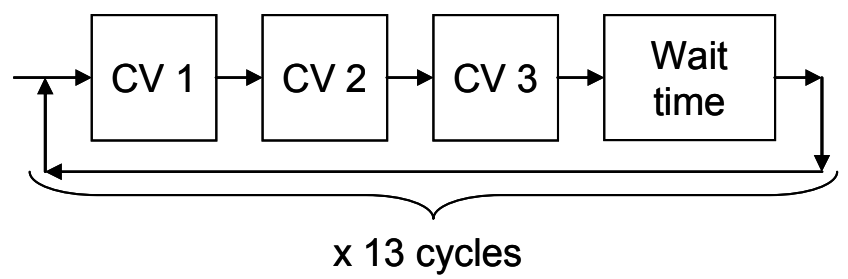

Figure 2. Experimental procedure followed for the $\mathrm{C}-\mathrm{V}$ measurements. Each cycle consists of 3 double sweep C-Vs, followed by a wait time. Illumination has been turned on only for cycles 3 and 4 .

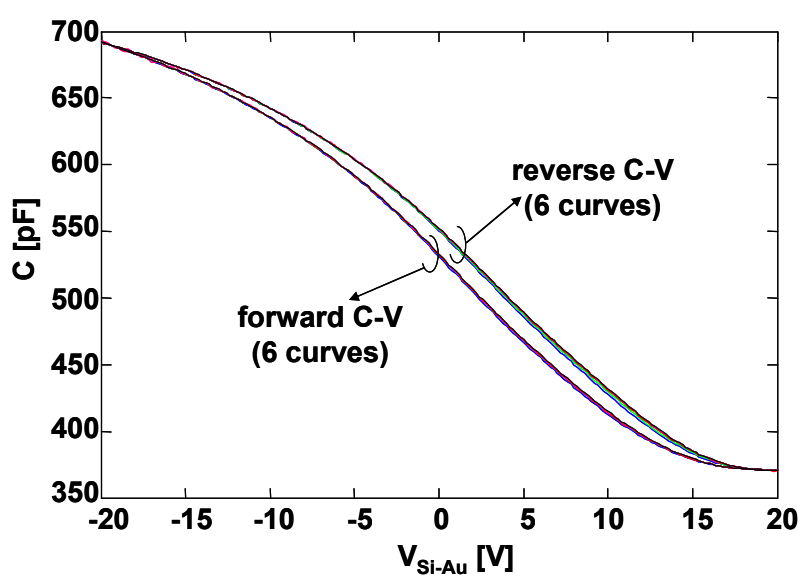

Figure 3. First 6 double sweep C-V performed in dark conditions (cycle 1 and cycle 2). The 6 curves are almost overlapping.

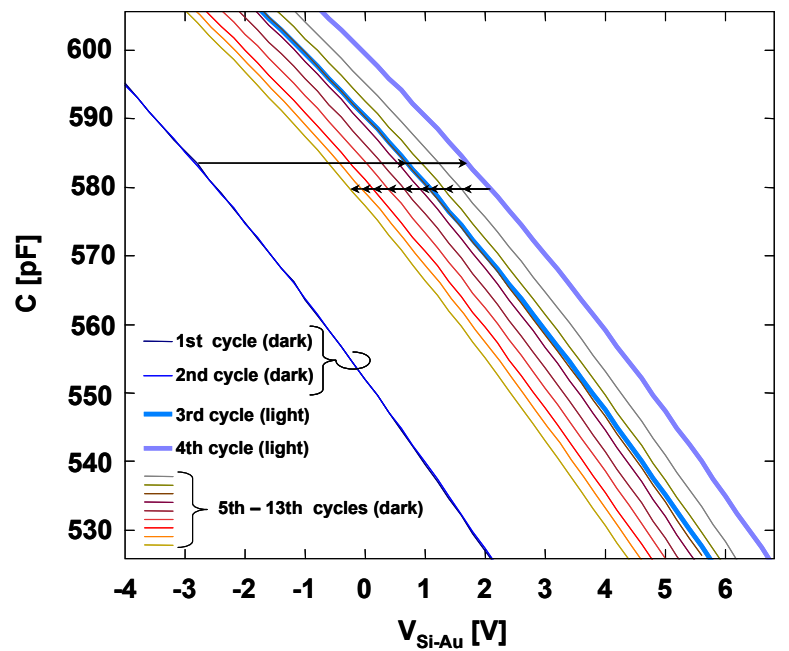

Figure 4. Zoom of the 3rd reverse C-V sweep of each cycle. When CVs are performed in dark, negligible changes occur (1st and 2nd cycle). Strong variation occurs, if the $\mathrm{C}-\mathrm{Vs}$ are performed under illumination (3rd and 4th cycle). If the C-Vs are performed again in dark, (cycles 5-13), the $\mathrm{C}-\mathrm{Vs}$ progressively move toward negative voltages. feature a hole mobility between 0.5 and $0.7 \mathrm{~cm}^{2} \mathrm{~V}^{-1} \mathrm{~s}^{-1}$ in ohmic region. $\mathrm{C}-\mathrm{V}$ measurements have been performed at $f=1 \mathrm{kHz}$ on a $2.8-\mathrm{mm}^{2}$ area capacitor, featuring the same vertical structure of the OTFT (see Fig. 1b); the pentacene layer around the gold pad was removed to achieve the desired capacitor area.

In the followings, we will refer as "bias" to the silicon (i.e. gate) to pentacene voltage.

\section{RESULTS}

\section{A. C-V and I-time measurements on organic capacitors}

Capacitors are useful tools to investigate device stability. In fact, fast interface states can be detected from the stretch of the $\mathrm{C}-\mathrm{V}$ measurement. The presence of slow traps (i.e. with response times comparable or larger than the $\mathrm{C}-\mathrm{V}$ measurement time) can be detected from the hysteresis of double sweep C-V measurement or even performing subsequent $\mathrm{C}-\mathrm{V}$ measurements. The presence of long-lived trapped charge can

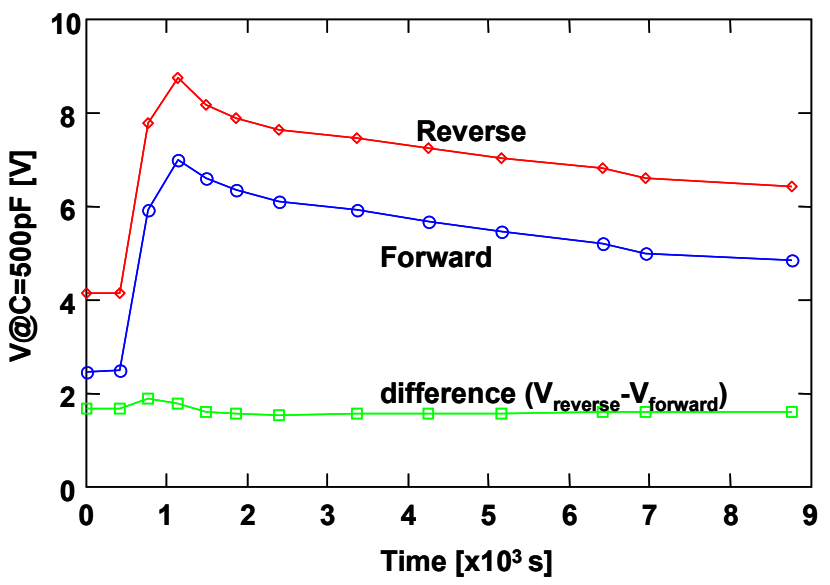

Figure 5. Voltages extrapolated from the third C-V of each cycle, at $\mathrm{C}=500 \mathrm{pF}$. The $\mathrm{C}-\mathrm{V}$ hysteresis (squares) is almost constant, regardless the shift on the $\mathrm{C}-\mathrm{V}$.

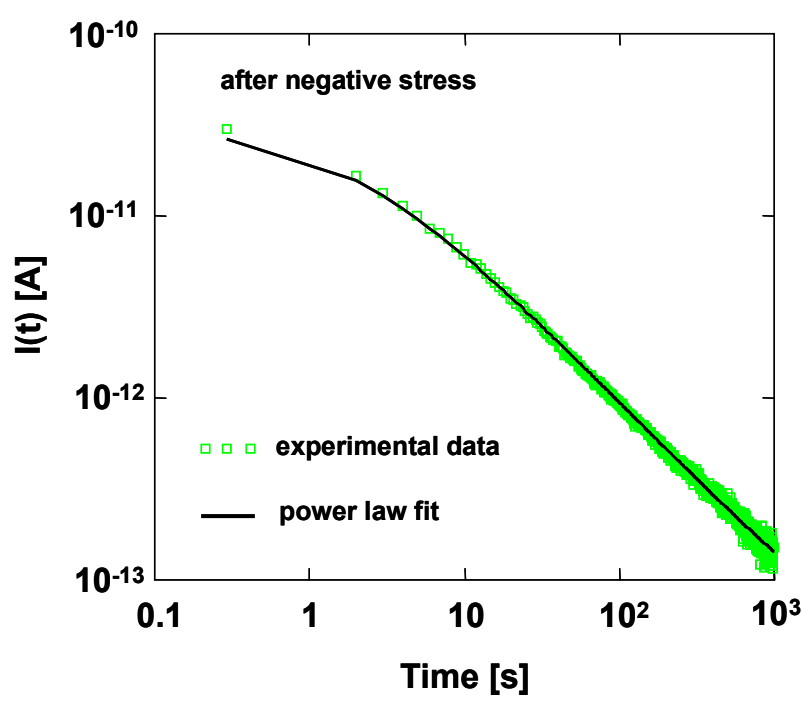

Figure 6. Evolution of the current measured after a $1000-\mathrm{s}-30-\mathrm{V}$ stress on a organic capacitor. Symbols are experimental data, while the solid line is the power-law fit (see Section IV). 
be detected also from the rigid shift of the $\mathrm{C}-\mathrm{V}$ after a constant stress. In particular, we performed the experimental procedure described in Fig. 2. Each measurement cycle consists of 3 consecutive double-sweep $\mathrm{C}-\mathrm{V}$ measurements. The first two cycles were performed in dark conditions and they are plotted in Fig 3. The $3^{\text {rd }}$ and $4^{\text {th }}$ cycle were performed under white light illumination, and all the other cycles were performed in dark. White light from a halogen light bulb was chosen in order to achieve a wide and continuous energy spectrum. While this choice does not allow us to establish any photon energy dependence, it is still useful to determine if charge trapping can be enhanced by light. The light power supply was chosen so that the optical power intensity was $0.1 \mathrm{~mW} / \mathrm{cm}^{2}$. A zoom of the transition region of the $\mathrm{C}-\mathrm{Vs}$ of the cycles 1 through 13 is plotted in Fig 4. For clarity, we show for each cycle only the third reverse $\mathrm{C}-\mathrm{V}$ sweep. In Fig 5, we plotted, for each cycle, the voltage required to achieve a capacitance of $500 \mathrm{pF}$, on the third forward $\left(\mathrm{V}_{\text {forward }}\right)$ and reverse $\left(\mathrm{V}_{\text {reverse }}\right) \mathrm{C}-\mathrm{V}$ sweep.

Remarkably, the C-Vs performed in dark (see Fig 3) show a hysteresis as large as $1.7 \mathrm{~V}$, indicating the presence of traps with response time comparable to the time taken by the $\mathrm{C}-\mathrm{V}$ measurement. Furthermore, despite the hysteresis, all the double sweep C-Vs of Fig 3 overlap each other.

On the contrary, when $\mathrm{C}-\mathrm{Vs}$ are taken under illumination, a large rightward shift occurs on both the forward and the reverse $\mathrm{C}-\mathrm{V}$ after the 3rd and 4th cycles (see Figs. 4 and 5), indicating that the double sweep $\mathrm{C}-\mathrm{V}$ measurement induced a net negative long-lived trapped charge. During cycles 5-13 (performed in dark) the $\mathrm{C}-\mathrm{V}$ begins to shift leftward indicating that the negative trapped charge slowly decreases.

Noticeably, the hysteresis in Fig. 5 is almost constant, regardless the shift on the $\mathrm{C}-\mathrm{V}$ curve (i.e. the negative trapped charge), suggesting that the traps responsible for the $\mathrm{C}-\mathrm{V}$ hysteresis are not the same traps responsible for the rigid rightward shift on the $\mathrm{C}-\mathrm{Vs}$.

We have also subjected the capacitor to a $1000-\mathrm{s}-30-\mathrm{V}$ constant negative bias stress. After that, we sampled the capacitor current for 1000 seconds (I-time measurement). The current evolution of the I-time measurement is shown in Fig. 6. The same procedure has been repeated using a $1000-\mathrm{s}+30-\mathrm{V}$ positive bias, with a similar evolution. As it will be discussed in Section IV, the capacitor current is excellently fitted with a power law, which decreases with time.

\section{B. Bias, light and temperature effects on OTFT}

While the experimental procedure of Fig. 2 provides some clues about charge trapping and traps density, it cannot be usefully exploited to perform more accurate investigations. In particular, the $\mathrm{C}-\mathrm{V}$ measurements take a considerable amount of time, hence its effect on the relax kinetics cannot be neglected, at least in the first 1000 seconds. Furthermore, the bias, which induces the trapped charge, is not constant during the $\mathrm{C}-\mathrm{V}$. Hence, we performed the experimental procedure shown in Fig. 7, for a much more accurate investigation on the role of light, temperature, and bias. In particular, we subjected the O-TFT to a 1000-s gate stress, with source and drain grounded (stress phase). The gate voltage during the stress phase was either $-30 \mathrm{~V}$ (negative stress) or $+30 \mathrm{~V}$ (positive stress) and the stress phase was performed in dark conditions. Just after the stress, we subjected the device to a relax phase, by applying no or moderate gate bias with source and drain grounded. The gate biases employed during the relax phases were either $0 \mathrm{~V},+5$ or $+10 \mathrm{~V}$, for the relax phases performed after the negative stress, and either $0,-5 \mathrm{~V}$ or $-10 \mathrm{~V}$ for the relax phases performed after the positive stress. The relax phase was performed either in dark or under illumination, using LEDs with different wavelengths. The LED biases were calibrated so that they emitted the same optical power. These relax phases were periodically interrupted to perform the $\mathrm{I}_{\mathrm{D}}-\mathrm{V}_{\mathrm{GS}}$ measurements. For these $\mathrm{I}_{\mathrm{D}}-\mathrm{V}_{\mathrm{GS}}$ measurements, the $\mathrm{V}_{\mathrm{GS}}$ sweep range was chosen so that the devices operated in the linear

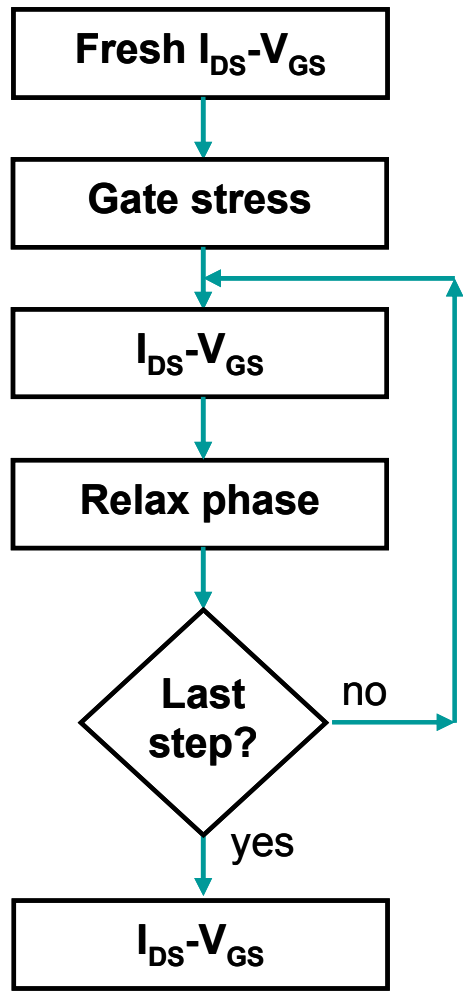

Figure 7. Experimental procedure used for the charge relaxation experiments described in section III-B.

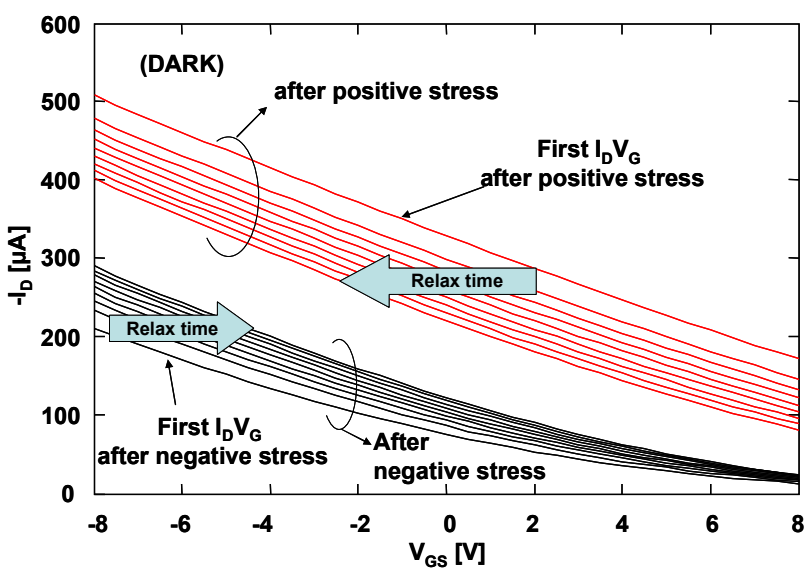

Figure 8. $\mathrm{I}_{\mathrm{D}}-\mathrm{V}_{\mathrm{GS}}$ curves measured during the charge relaxation experiments performed after the 1000-s gate stress. 
region and the corresponding $\mathrm{I}_{\mathrm{D}}$ was high enough to allow accurate and fast $I_{D}$ measurements, without inducing a strong impact on the relax kinetics. The total time taken from the $\mathrm{I}_{\mathrm{D}^{-}}$ $\mathrm{V}_{\mathrm{GS}}$ is less than 1 second.

Fig. 8 shows the $\mathrm{I}_{\mathrm{D}}-\mathrm{V}_{\mathrm{GS}}$ curves of a device taken during the relax phase after positive and negative stress, in dark. The $\mathrm{I}_{\mathrm{D}^{-}}$ $\mathrm{V}_{\mathrm{GS}}$ (in linear region) are almost parallel suggesting no modifications in the transconductance (and hence the carrier mobility), as already observed in [16],[23]. In the following, we define the gate voltage at which the drain current is $200 \mu \mathrm{A}$ as $V^{*}$. Fig. 9 shows the time evolution of the $V^{*}$ variation with respect to its initial value $\left(V^{*}(t)-V^{*}(0)\right)$, extrapolated from the $\mathrm{I}_{\mathrm{D}}-\mathrm{V}_{\mathrm{GS}}$ measured during the various relax phases. The lines are the fits, which will be discussed in Section IV.

Noticeably, if the relax phases is performed under $0-\mathrm{V}$ bias, the kinetics are independent on the illumination wavelength. Negative gate bias accelerates the relax kinetics but, again,

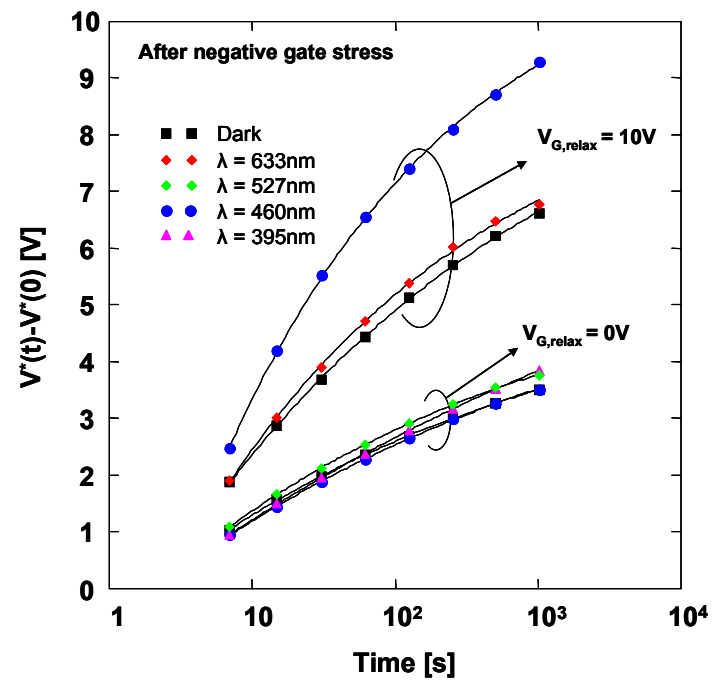

(a)

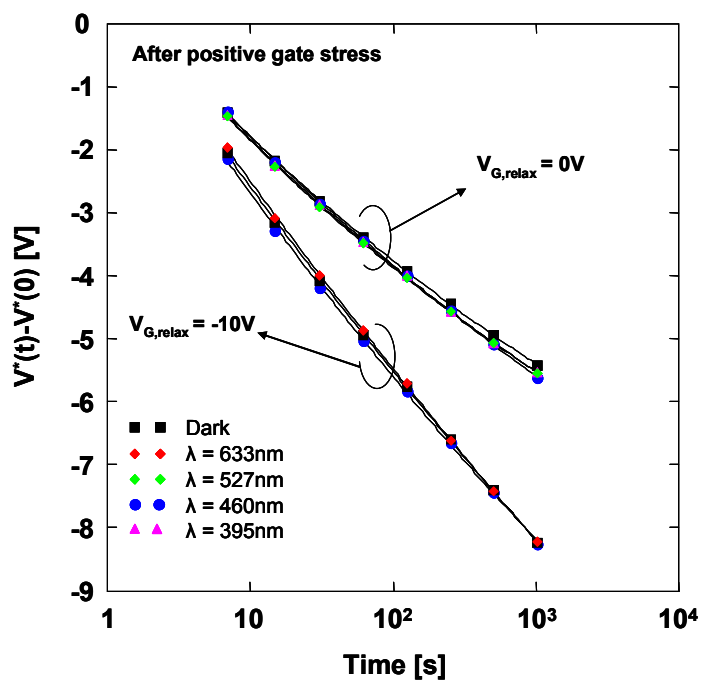

(b)

Figure 9. Variation of the $\mathrm{V}^{*}$ extrapolated from the $\mathrm{I}_{\mathrm{D}}-\mathrm{V}_{\mathrm{GS}}$ measured during the relax phases, under different illumination conditions: after the negative gate stress (a) and after the positive gate stress (b). there is no dependence on the illumination wavelength. On the contrary, light has a strong impact when a positive gate bias is applied during the relax phase after the negative stress.

\section{Effects of temperature}

We also analyzed the effects of temperature, performing the same relaxation experiments described in Fig. 7 at different temperatures, from $30^{\circ} \mathrm{C}$ to $60^{\circ} \mathrm{C}$. The maximum temperature was chosen so that any organic semiconductor degradation phenomena are avoided.

Remarkably, at least within our range, the temperature has a small impact on the transconductance. In fact, the transconductance variation between $30^{\circ} \mathrm{C}$ and $60^{\circ} \mathrm{C}$ is less than $4 \%$, hence, as a first approximation, its impact on the relax kinetics (if any) can be neglected. In particular, we found

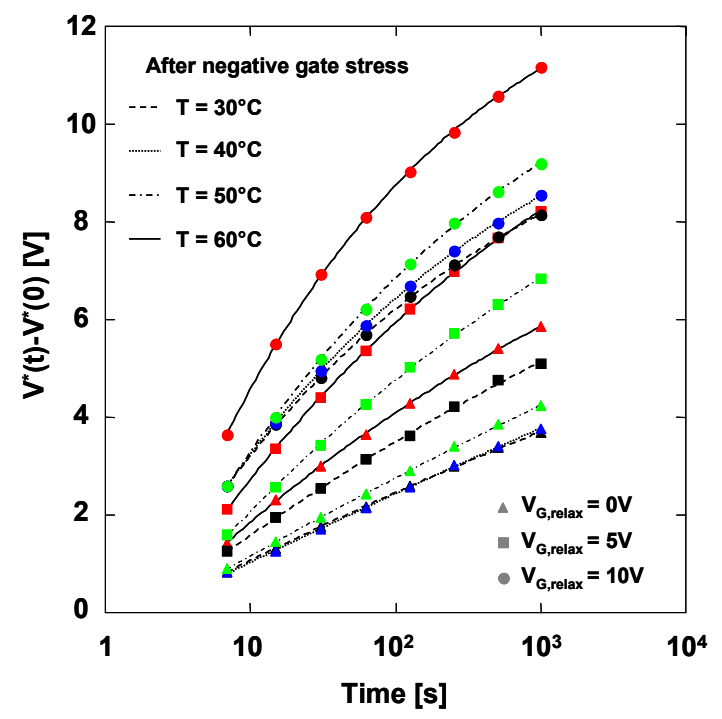

(a)

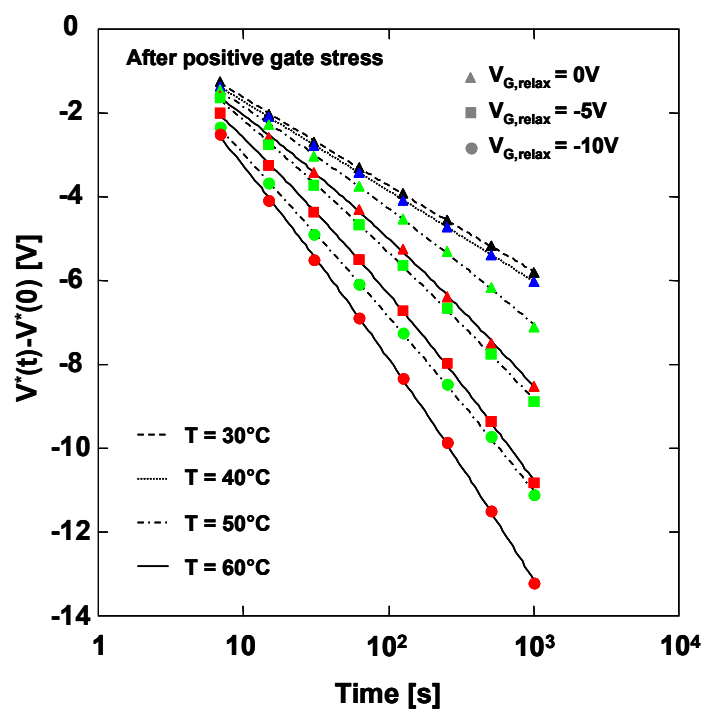

(b)

Figure 10. Variation of the $\mathrm{V}^{*}$ extrapolated from the $\mathrm{I}_{\mathrm{D}}-\mathrm{V}_{\mathrm{GS}}$ measured during the relax phases, at different temperatures: after the negative gate stress (a) and after the positive gate stress (b). 
$6.31 \mu \mathrm{S}$ and $6.56 \mu \mathrm{S}$ at $30^{\circ} \mathrm{C}$ and $60^{\circ} \mathrm{C}$, respectively. The results are summarized in Fig. 10. As expected, we found that the temperature accelerates the charge relaxation kinetics, especially under bias.

To avoid temperature effects on the stress and measurements, we also followed a different experimental setup. We have stressed the devices at a constant $-30 \mathrm{~V}$ gate bias, at $27^{\circ} \mathrm{C}$. Then, just after the stress, we measured the $\mathrm{I}_{\mathrm{D}}-\mathrm{V}_{\mathrm{GS}}$, characteristics. Later, we stored the device on a climatic chamber for 40 minutes, at a constant temperature ranging from $30^{\circ} \mathrm{C}$ to $60^{\circ} \mathrm{C}$. Due to the very small sample size and thermal mass, we expect that the thermal transient can be neglected over the total 40-minutes annealing time. During this annealing time, we left the device floating. After the 40minutes, we forced the device to cool-down to $27^{\circ} \mathrm{C}$ and we measured again the $\mathrm{I}_{\mathrm{D}}-\mathrm{V}_{\mathrm{GS}}$. In Fig. 11, we plot the ratio between the currents after and before annealing. The $\mathrm{I}_{\mathrm{D}}-\mathrm{V}_{\mathrm{GS}}$ curves measured before and after annealing are almost parallel, indicating that no mobility variation occurred and the threshold voltage variation was the responsible for the decreased current. Fig. 11 confirms that the charge relaxation kinetics is thermally activated, with an average activation energy of $213 \mathrm{meV}$.

\section{DiscUSSIONS}

The results of Figs. 3-11 indicate that moderately high biases induce charge trapping, whose sign depends on the bias polarity. In particular, with positive gate-to-pentacene bias, a net negative trapped charge contribution can be observed, while, if a negative gate-to-pentacene bias is employed, positive charge is trapped.

In the literature some authors reported charge trapping in organic TFT, both polymer and small-molecule based [13][16]. In principle charge can be trapped at: the bulk of the oxide, at the pentacene/oxide interface, in the bulk of the pentacene layer. Due to the relatively small electric field, we argue that the charge trapped in the bulk of the $\mathrm{SiO}_{2}$ is negligible. In fact, during our stress and relax kinetics, the oxide electric field is in the range of $0.7-2.1 \mathrm{MV} / \mathrm{cm}$, which is a very low value to achieve appreciable charge injection [24]-

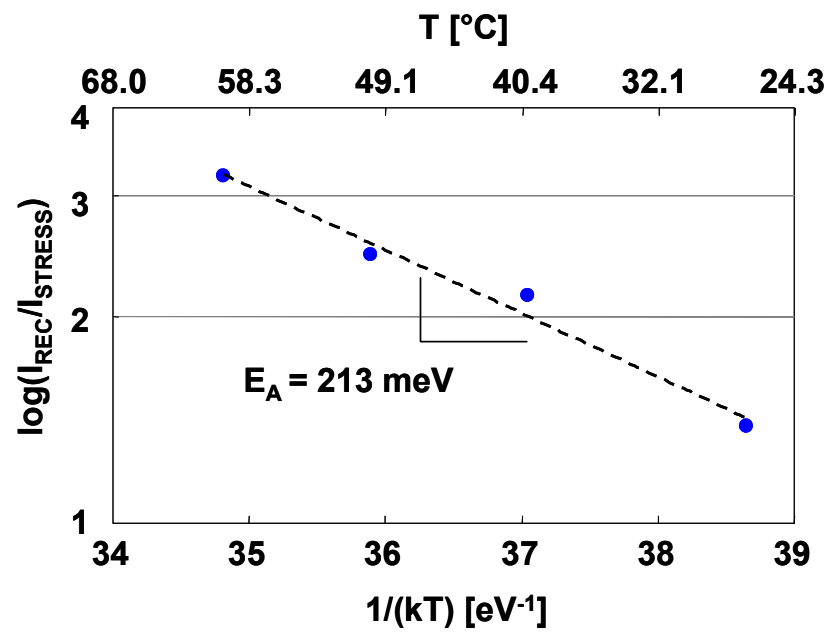

Figure 11. Arrhenius plot of the drain current recovery induced by a 40min annealing performed after the negative stress.
[25]. Traps (or even border traps) at the dielectric/pentacene interface might account for the hysteresis measured during the $\mathrm{C}-\mathrm{V}$ measurements. Finally, some authors reported about trap in the organic semiconductor [17],[26]-[27] or bias-induced charged defects [27], which, in turn, move in the organic semiconductor, inducing long-lived threshold voltage variations.

In the following we will discuss about the combined effects of light and bias (Section IV-A), the temperature effects (Section IV-B) and we will provide a first order model for the relaxation kinetics (Section IV-C).

\section{A. Light and bias effects}

The C-V measurements (see Figs. 3-5) clearly highlight the presence of traps featuring different response times. On one hand, we observed a hysteresis (see $\mathrm{V}_{\text {forward }}-\mathrm{V}_{\text {reverse }}$ of Fig 5), which is almost constant regardless the measurement condition (dark or under illumination). Taking into account the $\mathrm{C}-\mathrm{V}$ measurement and sampling times, we argue that the response time of the traps responsible for the $\mathrm{C}-\mathrm{V}$ hysteresis is within 10-100 seconds. In fact, traps faster than the sampling-time would only stretch-out the $\mathrm{C}-\mathrm{V}$ curve, whereas traps much slower than the total $\mathrm{C}-\mathrm{V}$ measurement-time would not be affected by the $\mathrm{C}-\mathrm{V}$ measurement, hence, they would not give any contribution on the total trapped charge, which is reflected on the $\mathrm{C}-\mathrm{V}$ position.

On the other hand, if the double-sweep $\mathrm{C}-\mathrm{V}$ is performed under illumination, both the forward and the reverse curves are shifted rightward (see $V_{\text {forward }}$ and $V_{\text {reverse }}$ in Fig 5), indicating that negative charge has been trapped under the combined action of light and bias. Remarkably, the presence of this trapped charge does not affect the hysteresis width, which is constant. Once the light is removed, a very slow relax kinetics can be measured on the $V_{\text {forward }}$ and $V_{\text {reverse }}$ (see Fig 5, after $1140 \mathrm{~s})$. The timescale of this kinetics is much slower than the $\mathrm{C}-\mathrm{V}$ measurement time and from Fig. 5 we argue that these trap, which are responsible for the almost rigid shift of both $\mathrm{V}_{\text {forward }}$ and $\mathrm{V}_{\text {reverse }}$, have a response time at least two orders of magnitude slower than the traps responsible for the $\mathrm{C}-\mathrm{V}$ hysteresis. This explains why the hysteresis is almost unaffected by the particular value of $V_{\text {forward }}$ or, equivalently, $\mathrm{V}_{\text {reverse: }}$ by the time the full double-sweep $\mathrm{C}-\mathrm{V}$ is performed $(120 \mathrm{~s})$, very small net charge is trapped in dark, and the position of the forward-sweep $\mathrm{C}-\mathrm{V}$ relative to the reversesweep $\mathrm{C}-\mathrm{V}$ depends almost only on the traps with response time within 10-100s.

From the $\mathrm{C}-\mathrm{V}$ measurements, we also argue that light mostly enhances negative charge trapping. Negative charge trapping (or equivalently, hole neutralization) occurs when the gate-to-pentacene bias is positive (see position of forwardsweep $\mathrm{C}-\mathrm{V}$ with respect the reverse-sweep $\mathrm{C}-\mathrm{V}$ ). This also confirmed by Fig 9a. In fact, from Fig. 9a we noticed that trapped hole neutralization is strongly enhanced under blue light if a positive bias is applied.

From the data of Fig. 5, we estimate that an interface trap density of $2.6 \cdot 10^{11} \mathrm{~cm}^{-2}$ can account for the $1.7-\mathrm{V}$ hysteresis, while the $4.6 \mathrm{~V}$ shift on both the forward and reverse $\mathrm{C}-\mathrm{V}$ is induced by a negative trapped charge density of $7.0 \cdot 10^{11} \mathrm{~cm}^{-2}$ (if 
all the charges are supposed concentrated at the $\mathrm{SiO}_{2} /$ pentacene interface). In about two hours, only half of these traps are effectively discharged.

Coming now to the stress, we argue that, due to the electric field direction, most of the trapped charges are located near the pentacene $/ \mathrm{SiO}_{2}$ interface. When a high enough positive bias is applied, electrons are trapped. Photons with enough energy increase the effects of positive bias, because of the excess of photogenerated electrons. In fact, it is well known [28] that photons with energy larger than the energy gap can photogenerate electron-hole pairs, which may either be trapped or they may neutralize the trapped holes. Conversely, if a high enough negative bias is applied, an excess of positive charge is trapped. At least with our optical intensity $\left(\sim 0.1 \mathrm{~mW} / \mathrm{cm}^{2}\right)$, light has a minimal impact because holes are majority carrier and the excess of photogenerated holes is negligible. Hence, there is also negligible light wavelength dependence under negative bias.

In the same way, light accelerates positive charge neutralization if a positive bias is applied. In fact photogenerated electrons drift toward the interface under the action of the positive bias, neutralizing the trapped holes [16]. Again, at least under low level of illumination intensities, light does not accelerate electron neutralization under negative bias, because the excess of photogenerated holes is negligible with respect to the equilibrium hole density value.

The effect of light under positive bias explains why the C-V measurements performed under white light feature a strong

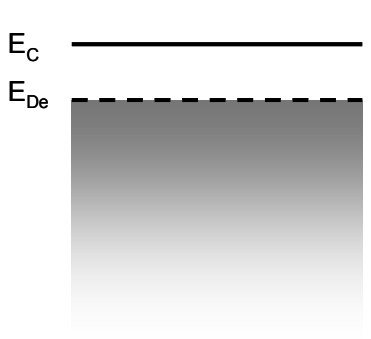

(a)

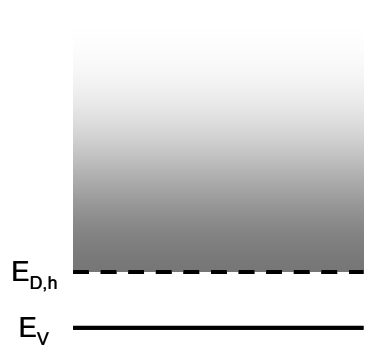

(c)

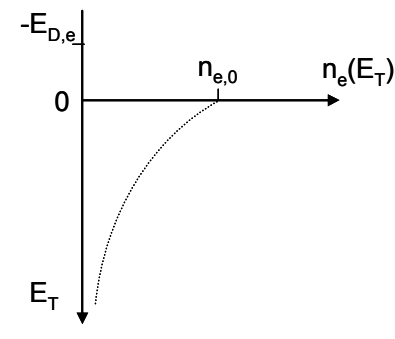

(b)

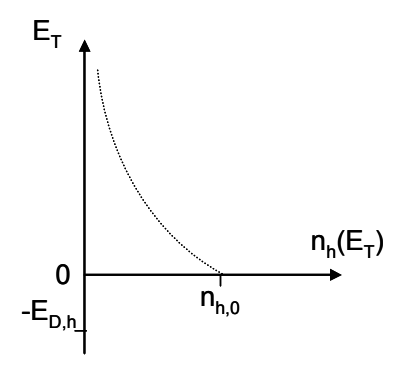

(d)
Figure 12. Visual representation of the electron trap density below the conduction band (LUMO band). Darker zones correspond to higher density (a). Sketch of the exponential energy trap density assumed for electrons in Section IV-C (b). Visual representation of the hole trap density above the valence band (HOMO band). Darker zones correspond to higher density (c). Sketch of the exponential energy trap density assumed for holes in Section IV-C (d) rightward shift. In fact, when the measurements are performed in dark, the positive and negative gate biases induce almost the same absolute value of $V^{*}$ variation (around $4 \mathrm{~V}$ in 30s, see Fig. 9). However, when a positive relax bias is applied under light (e.g. $\lambda=460 \mathrm{~nm}$ in Fig 9), a $6-\mathrm{V} V^{*}$ variation occurs, whereas the $V^{*}$ variation with negative relax bias is $-4 \mathrm{~V}$, independent of the illumination condition.

\section{B. Effects of temperature}

Figs. 10-11 indicate that temperature has a strong impact on the charge detrapping kinetics, especially if we consider the very small temperature variation (only $30^{\circ} \mathrm{C}$ ). The temperature might accelerate the relaxation kinetics in several ways. First, it is well known that the time to escape from a trap exponentially depends on the temperature [25],[29]. Secondly, the mobility of charged mobile defects might be increased by the temperature. The bias might also accelerate the relaxation kinetics adding a drift contribution to the thermal-activated diffusion of mobile charged defects, which move away from the interface toward the source/drain contacts.

The 213-meV activation energy extrapolated in Fig. 11 perfectly correlates with the $0.2-\mathrm{eV}$ activation energy associated with the electron transfer from/to hydrogenoid species [30]. These species may derive either from $\mathrm{H}$ atoms used for dangling bond passivation or from $\mathrm{H}_{2} \mathrm{O}$ contaminants within the parylene encapsulation layer, which eventually migrate toward the $\mathrm{SiO}_{2}$ /pentacene layer during aging.

\section{Relax kinetics}

According with literature [15], all the relax kinetics of Figs. 9-10, fit with a power law. In particular, we found that our experimental data excellently fit with the following power law:

$$
\Delta V^{*}(t)=V^{*}(t)-V^{*}(0)=C\left[1-\left(\frac{t}{t_{0}}\right)^{\gamma}\right] .
$$

In the following we give a first order model that allows to quantitatively estimate the mean trap energy level.

In principle, the trap energetic distribution within the pentacene layer might be position dependent. Furthermore, $V^{*}$ also depend on the actual trapped charge position. For sake of simplicity, we will assume that all the trapped charges are located near the interface between $\mathrm{SiO}_{2}$ and pentacene. Of course, this is only a first order approximation, since traps might also be located in the bulk of the pentacene, as already stated before and as it has been also reported in [31]. Still, the calculated interface trap density might be viewed as an equivalent trap density, which accounts both for the interface and for the pentacene bulk trap density.

According with several papers in literature, we expect that traps in the in the polycrystalline pentacene are energetically distributed between the HOMO and LUMO energy level (see Figs. 12a and 12b). In particular, we assume that there is an exponential distribution of deep and slow traps starting at depth $E_{D, e}$ below the LUMO and it exponentially fades with a energy constant $U$. Following these assumptions, we write the trap 
density per unit area and per unit energy as (assumed concentrated at the $\mathrm{SiO}_{2} /$ pentacene interface):

$$
d_{e}\left(E_{T}\right)=d_{e, 0} \cdot e^{-E_{T} / U},
$$

Where $E_{T}$ is the trap depth with respect to $E_{D, e}$ (see Fig. $12 \mathrm{~b})$. Of course, (2) is only a first order approximation and holds only for a limited $E_{T}$ range. A similar distribution was hypothesized in [32].

Shallow traps are supposed energetically concentrated at $E_{D, e}$ and we suppose that such shallow traps quickly exchange electrons/hole in time much shorter than 2 seconds, i.e., the time elapsed by the end of the stress pulse and the beginning of the I-time sampling. For this reason, we neglect the contribution of the shallow traps in the hysteresis and in the slow C-V curve displacement during the relax period. The same hypotheses and considerations holds true for the HOMO level (see Figs. 12c and 12d).

In a first approximation, we also assume that, after the positive stress, all the traps energetically located below the Fermi level are filled with an electron, i.e. the filled trap density per unit area and per unit energy is $n_{e}\left(E_{T}\right)=d_{e}\left(E_{T}\right)$ :

We suppose the emission time $\tau$ of a trap located at energy $E_{T}+E_{D}$ below the LUMO (or above the HOMO) exponentially depends on the trap depth, i.e.,

$$
\tau\left(E_{T}\right)=\tau_{0} \cdot e^{E_{T} /(K T)},
$$

Where $\tau_{0}$ depends also on the temperature, bias and $E_{D, e}$.

Now, at the time $t$, the number of detrapped electrons is:

$$
N_{e}(t)=\int_{0}^{E_{T}(t)} n_{e}\left(E_{T}\right) d E_{T},
$$

Where $E_{T}(t)$ is found solving (3) for $E_{T}$.

Using (2) and (4) we find:

$$
N_{e}(t)=\frac{d_{e, 0}}{U}\left[1-\left(\frac{t}{\tau_{0}}\right)^{-\frac{K T}{U}}\right] .
$$

Assuming the effect of $N_{e}(t)$ as if it were concentrated at the $\mathrm{SiO}_{2} /$ pentacene interface, the corresponding $\Delta V^{*}(t)$ describing the relax kinetics after the positive gate stress is:

$$
\Delta V^{*}(t)=-\frac{q}{C_{o x}} \cdot \frac{d_{e, 0}}{U}\left[1-\left(\frac{t}{\tau_{0}}\right)^{-\frac{K T}{U}}\right],
$$

which has the same form of (1).

The same procedure holds true for the case of trapped holes $\left(N_{h}\right)$ after a negative stress. Of course, in this case the resulting sign is opposite to (6).

Our results indicate that $U$ is within the range of 150$400 \mathrm{meV}$.

Incidentally, the relax current measured on a capacitor also fits with a power law:

$$
i(t)=i_{0}\left(\frac{t}{t_{i}}+1\right)^{-D}+i_{k},
$$

In (7), the first term is the transient component, responsible for the current kinetics at least for the first 1000 seconds. On the contrary the constant $i_{k}$ accounts for both the capacitor and the instrumentation/cables/setup leakage, which do not play any role in the charge trapping kinetics. The same constant might also account for a much slower kinetics (such as those induced by the motion of hydrogenoid species), which induces negligible effects at least in the first $1000 \mathrm{~s}$, but it could play a key-role if much longer timescales are considered. The constant $i_{k}$ will considered equal to zero hereafter.

After simple calculation (see appendix) we derive the trap energetic distribution:

$$
n\left(E_{T}\right)=\frac{i_{0} \cdot \tau_{0}}{q \cdot K T} \cdot \frac{e^{E_{T} /(K T)}}{\left(\frac{\tau_{0}}{t_{i}} e^{E_{T} /(K T)}+1\right)} .
$$

In our fits, we found that $\tau_{0} \cong t_{l}=2 \mathrm{~s} \pm 0.3 \mathrm{~s}$, hence, if $E_{T} /(K T)$ is high enough, the right term denominator of (8) can be approximated as $\left(\left(t_{0} / t_{i}\right) e^{E_{T} /(K T)}\right)^{D}$. Using this approximation, (8) has the form of (2). In other words, if we assume that (3) holds, and if the measured current fits with (7), then the $n\left(E_{T}\right)$ has, with good approximation, the exponential form hypothesized in (2).

\section{APPENDIX}

It is straightforward to derive the following result from (4):

$$
i(t)=\frac{d Q}{d t}=q \frac{d N\left(E_{T}(t)\right)}{d t}=q \cdot n_{e}\left(E_{T}\right) \frac{d E_{T}(t)}{d t},
$$

From (9), one can obtain:

$$
n\left(E_{T}\right)=\frac{i(t)}{q} \cdot \frac{1}{\frac{d E_{T}(t)}{d t}}=\frac{i(t)}{q} \cdot \frac{t}{K T},
$$

Now, using (8) and substituting $\mathrm{t}$ with (3), we find $n\left(E_{T}\right)$ :

$$
n\left(E_{T}\right)=\frac{i_{0} \cdot \tau_{0}}{q \cdot K T} \cdot \frac{e^{E_{T} /(K T)}}{\left(\frac{\tau_{0}}{t_{i}} e^{E_{T} /(K T)}+1\right)} .
$$

\section{CONCLUSIONS}

In this work, we showed the combined effects of bias, light and temperature on organic thin-film transistors. Relatively high biases induce a noticeable charge trapping, which manifests itself as a rigid shift of the transfer characteristics. Such shift can be larger than $10 \mathrm{~V}$ if a $\pm 30 \mathrm{~V}$ bias is applied for 1000 seconds. However, no bias-induced effects can be appreciated on the device transconductance. 
Light enhances both the electron trapping and hole neutralization kinetics, if a positive bias is applied after the negative gate-to-source stress, whereas the effects of light are negligible under zero or negative bias after the positive gate-tosource stress. Still, we do not exclude that with a much higher optical intensity light could also enhance hole trapping or electron neutralization.

Temperature increases the charge detrapping rate, and the calculated activation energy suggests that the motion of hydrogenoid species may have a strong contribution on the relaxation kinetics.

Finally, we presented a first order model, which provides excellent fits of the charge relaxation kinetics, in all the operating conditions.

\section{REFERENCES}

[1] D.J., Gundlach, C.-C., Kuo, S.F. Nelson, and T.N. Jackson, "Organic thin film transistors with field effect mobility $>2 \mathrm{~cm} 2 / \mathrm{V}-\mathrm{s}$," 57 th Annual Device Research Conference Digest, 1999, pp.164-165, 1999.

[2] S. K. Park, C.-C. Kuo, J.E. Anthony, and T.N. Jackson, "High mobility solution-processed OTFTs," IEEE International Electron Devices Meeting, 2005. IEDM Technical Digest, pp. 4, 5-7 Dec. 2005.

[3] C.-C. Kuo; M.M. Payne, J.E. Anthony, and J.E. Jackson, "TES anthradithiophene solution-processed OTFTs with $1 \mathrm{~cm}^{2} / \mathrm{V}$-s mobility," IEEE International Electron Devices Meeting, 2004. IEDM Technical Digest. , pp. 373-376, 13-15 Dec. 2004.

[4] D.J. Gundlach,H. Klauk, C.D. Sheraw, C.-C. Kuo; J.-R. Huang; and T.N. Jackson, "High-mobility, low voltage organic thin film transistors,"

[5] Z. Bao, A. J. Lovinger, and A. Dodabalapur ,"Organic field-effect transistors with high mobility based on copper phthalocyanine," Appl. Phys. Lett. 69, 3066 (1996).

[6] R. Parashkov, E. Becker, G. Ginev, T. Riedl, H.-H. Johannes, and W. Kowalsky, "All-organic thin-film transistors made of poly(3butylthiophene) semiconducting and various polymeric insulating layers," J. Appl. Phys. 95, 1594 (2004).

[7] Fang-Chung Chen, Chih-Wei Chu, Jun He, and Yang Yang, "Organic thin-film transistors with nanocomposite dielectric gate insulator," Appl. Phys. Lett. 85, 3295 (2004)

[8] H. Klauk, M. Halik, U. Zschieschang, G.Schmid, and W. Radlik, "Highmobility polymer gate dielectric pentacene thin film transistors," J. Appl. Phys. 92, 5259 (2002).

[9] T.N. Jackson, "Organic thin film transistors-electronics anywhere," International Semiconductor Device Research Symposium, 2001, pp.340-343, 2001.

[10] K. Kudo, "Recent progress on organic thin film transistors and flexble display applications," Nanotechnology Materials and Devices Conference, 2006. NMDC 2006. IEEE, vol.1, pp.290-291, 22-25 Oct. 2006.11 IRPS 09

[11] C. Pannemann, T. Diekmann, and U. Hilleringmann, "On the degradation of organic field-effect transistors," Microelectronics, 2004. ICM 2004.

[12] Y. Qiu, Yuanchuan Hu, Guifang Dong, Liduo Wang, Junfeng Xie, and Yaning Ma, " $\mathrm{H}_{2} \mathrm{O}$ effect on the stability of organic thin-film field-effect transistors," Appl. Phys. Letters, (83), 8,2003, pp. 1644-1646.

[13] Hsiao-Wen Zan, and and Shin-Chin Kao "The Effects of Drain-Bias on the Threshold Voltage Instability in Organic TFTs ," IEEE Elec. Dev. Lett, vol 29, no. 2, Feb. 2008, pp .155-157.
[14] C. Erlen, F. Brunetti, P. Lugli, M. Fiebig, S. Schiefer, and B. Nickel, "Trapping Effects in Organic Thin Film Transistors," in Proc. of Sixth IEEE Conference of Nanotechnology, June 2006, pp. 82-85.

[15] A. Salleo, F. Endicott, and R. A. Street, "Reversible and irreversible trapping at room temperature in pol(thiophene) thin-film transistors,", Appl. Phys. Lett. Vol. 86, 263505, 2005.

[16] M. Debucquoy, S. Verlaak, S. Steudel, K. Myny, J. Genoe, and P. Heremans, "Correlation between bias stress instability and phototransistor operation of pentacene thin-film transistors," Appl. Phys. Lett. 91, 103508 (2007).

[17] Jae-Hoon Lee, Sang-Geun Park, Sang-Myeon Han, Min-Koo Han, and Kee-Chan Park ,"New PMOS LTPS-TFT pixel for AMOLED to suppress the hysteresis effect on OLED current by employing a reset voltage driving," Solid-State Electronics 52 (2008) 462-466

[18] Z. Tang, M.S. Park, S.H. Jin, C.R. Wiem, "Drain bias dependent bias temperature stress instability in a-Si:H TFT,", Solid-State Electronics, vol. 53 (2009), pp. 225-233.

[19] Jae-Hoon Lee, Woo-Jin Nam, Kwang-Sub Shin, and Min-Koo Han, "Hysteresis phenomenon of hydrogenated amorphous silicon thin film transistors for an active matrix organic light emitting diode," Journal of Non-Crystalline Solids vol. 352 (2006) pp. 1719-1722.

[20] Taek Ahn, Hye Jung Suk, Jongchan Won, Mi Hye Yi, "Extended lifetime of pentacene thin-film transistor with polyvinyl alcohol (PVA)/layered silicate nanocomposite passivation layer," Microelectronic Engineering, vol. 86. (2009), pp 41-46

[21] Juan Li, Chunya Wu, Jianpin Liu, Shuyun Zhao, Zhiguo Meng, Shaozhen Xiong, and Lizhu Zhang, "A new instability phenomenon in microcrystalline silicon thin film transistors", Journal of Non-Crystalline Solids, vol. 352 (2006) 1715-1718.

[22] Bong-Hyun You, Jae-Hoon Lee, and Min-Koo Han, "Polarity Balanced Driving Scheme to Suppress the Degradation of Vth in a-Si:H TFT Due to the Positive Gate Bias Stress for AMOLED," IEEE journ. Display Technology, vol. 3, no. 1, mar. 2007, pp. 40-44.

[23] Tae Ho Kim, Chung Kun Song, Jin Seong Park, and Min Chul Suh, "Constant Bias Stress Effects on Threshold Voltage of Pentacene ThinFilm Transistors Employing Polyvinylphenol Gate Dielectric", IEEE Elec. Dev. Lett. vol. 28, nO. 10, Oct. 2007, pp. 874-876.

[24] M. Lenzlinger, E.H. Snow, "Fowler-Nordheim tunnelling into thermally grown $\mathrm{SiO}_{2}$ ”, J. Appl. Phys., Vol. 40, pp. 278-283, 1969.

[25] P. Hesto, in Instability on Silicon Devices, Ed. G. Barbottin and A. Vapaille, North Holand, Vol. 1, 1986.

[26] John E. Northrup and Michael L. Chabinyc, "Gap states in organic semiconductors: Hydrogen- and oxygen-induced states in pentacene," Phys. Rev. B 68, 041202(R) (2003).

[27] D.V. Lang, X. Chi, T. Siegrist, A.M. Sergent, and A. P. Ramirez, "BiasDependent Generation and Quenching of Defects in Pentacene," Phys. Rev. Lett., vol. 93, no. 7, 076601, 2004.

[28] Tse Nga Ng, M.L. Chabinyc, R.A. Street, and A. Salleo, "Bias Stress Effects in Organic Thin Film Transistors," 45th annual. IEEE International Reliability physics symposium, 2007. proceedings., pp.243-247, 15-19 April 2007.

[29] W. B. Jackson, J. M. Marshall, and M. D. Moyer, "Role of hydrogen in the formation of metastable defects in hydrogenated amorphous silicon", Phys. Rev. B 39, 1164-1179 (1989).

[30] K. Vanheusden, S. P. Karma, R. D. Pugh, W. L. Warren, D.M Fleetwood, and R. A. B. Devine, "Thermally activated electron capture by mobile proton in $\mathrm{SiO}_{2}$ thin films", Appl- Phy- Lett. 72, pp. 28, 1998.

[31] V. Nádaždy, R. Durný, J. Puigdollers, C. Voz, S. Cheylan, and M. Weis, "Defect states in pentacene thin films prepared by thermal evaporation and Langmuir-Blodgett technique," Journal of Non-Crystalline Solids, Vol. 354, May 2008, pp 2888-2891.

[32] A. Rose, "Space-Charge-Limited Currents in Solids", Physical Review, Vol. 97, no. 6, Mar. 1955, pp 1538-1544. 